\title{
The Value of Imaging Methods in the Diagnosis of the Renal Cell Carcinoma Combined with Renal Tuberculosis - Case Report
}

\author{
Tania Tsocheva $^{1 *}$, Regina Djerassi ${ }^{1}$, Aleksandrina Vlahova $^{2}$, Mila Liubomirova $^{1}$, Boris $^{*}$ Bogov $^{1}$ \\ ${ }^{1}$ Hospital Alexandrovska, Clinic of Nephrology, 1 "St. G. Sofiiski" str., Sofia 1431, Bulgaria; ${ }^{2}$ Hospital Alexandrovska, \\ Department of Pathoanatomy, Sofia 1431, Bulgaria
}

\author{
Citation: Tsocheva T, Djerassi R, Vlahova A, \\ Liubomirova M, Bogov B. The Value of Imaging \\ Methods in the Diagnosis of the Renal Cell \\ Carcinoma Combined with Renal Tuberculosis - \\ Case Report. OA Maced J Med Sci. 2013 Dec \\ 15; 1(1):90-92 \\ http://dx.doi.org/10.3889/oamjms.2013.018 \\ Key words: Granulomatous inflammation; \\ epithelioid histiocytes; multinucleated giant \\ cells; renal cell carcinoma; case report. \\ "Correspondence: Dr. Tania Tsocheva. Clinic \\ of Nephrology, University Hospital" \\ Alexandrovska", 1 "St.G. Sofiiski"str., 1431 \\ Sofia, Bulgaria. Tel: ++35929230214. E-mail: \\ trc_s@abv.bg \\ Received: 24-Oct-2013; Revised: 19-Nov- \\ 2013; Accepted: 27-Nov-2013; Online first: \\ 02-Dec-2013 \\ Copyright: (c) 2013 Tsocheva T. This is an \\ open-access article distributed under the terms \\ of the Creative Commons Attribution License, \\ which permits unrestricted use, distribution, \\ and reproduction in any medium, provided the \\ original author and source are credited. \\ Competing Interests: The authors have \\ declared that no competing interests exist.
}

\begin{abstract}
We describe a case of renal cell carcinoma which causes difficulties to set diagnosis with imaging methods. The histological examination revealed renal cell carcinoma associated with epithelioid cell granuloma with caseous necrosis. Granulomatous inflammation is characterized by the accumulation of epithelioid histiocytes and multinucleated giant cells. The cause of granuloma formation can be infectious or non-infectious. Granulomatous response in renal cell carcinoma is rare. This case shows that for accurate diagnosis for suspected tumor is necessary to be used combination of complex imaging - triplex ultrasound, CEUS, CT and histological examination.
\end{abstract}

\section{Introduction}

Granulomatous inflammation is characterized by the accumulation of epithelioid histiocytes and multinucleated giant cells. Granulomatous response in renal cell carcinoma is rare. It may happen due to Tcell mediated response against antigens, expressed by the tumor or the presence of chronic inflammatory process like the tuberculosis. Coexistence of tuberculosis and carcinoma in the same organ is rare, particularly in the kidney [1]. We present a case of a 61- year old man with renal cell carcinoma and tuberculosis of the same kidney.

\section{Case report}

We report on a 61 -years- old man who presented with dull flank pain without irradiation. $\mathrm{He}$ had no dysuria, hematuria, nor fever. He reported raised BP to 140/90 $\mathrm{mm} \mathrm{Hg}$ for the past 2 weeks, usual values around 110/70 $\mathrm{mm} \mathrm{Hg}$.

The physical examination showed no significant pathological findings. Laboratory analysis hemoglobin, red blood cells, ESR within the normal limits, white blood cells - 17,000 without left shift, creatinine, urea were within the normal limits. Urine sediment: 5-6 white blood cells/high power field.

Ultrasound examination showed normal utrasound images of liver, gall bladder, spleen, left kidney, pancreas. Prostate was normal-sized, homogeneous. Right kidney was $118 \mathrm{~mm}$ longitudinal size, $12 \mathrm{~mm}$ parenchyma. There was a heterogeneous formation 33/35 with hyperechogenic contour surrounded by uneven hypoechogenic capsula. 


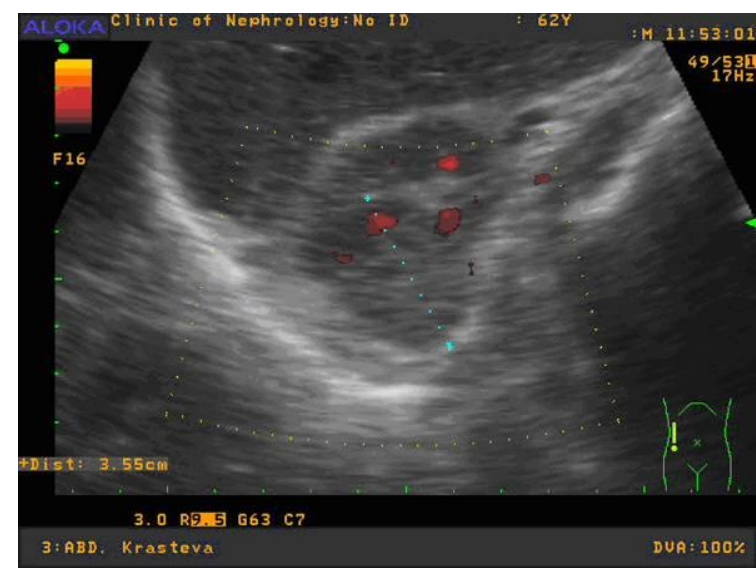

Figure 1: Color Doppler - the picture shows mainly peripheral blood vessels.

The Color Doppler demonstrated poorly vascularised formation with mainly peripheral vascularization (Fig. 1). There was a small arteriovenous fistula, visible with pulse Doppler, in one of the sections. The Doppler curves showed different peak velocity in the tumor vessels with irregular Doppler curve (Fig. 2).

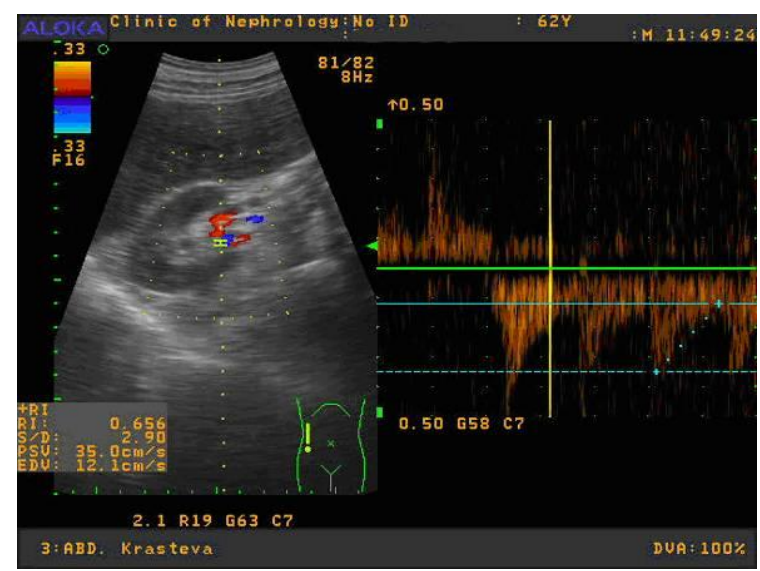

Figure 2: Doppler curves - the Dopler curve is irregular with different peak velocity in the tumor vessels.

We performed contrast enhanced ultrasound (CEUS) for better evaluation of blood vessels in the formation, using SonoVue contrast agent. The wash in phase was slow.

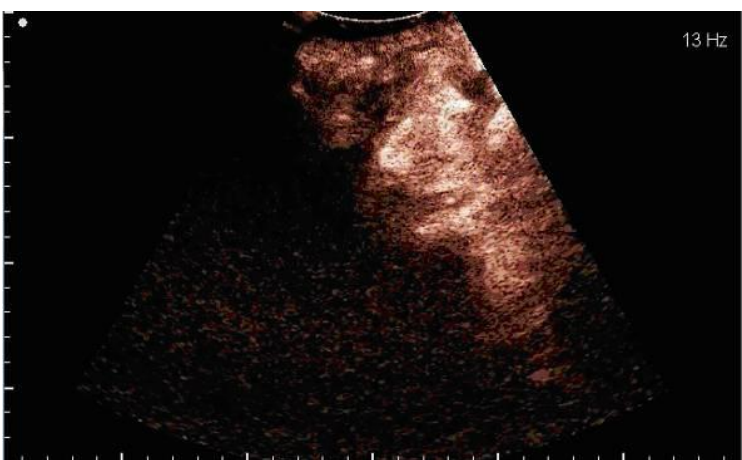

Figure 3: CEUS - contrast distribution resembles to angiomyolipoma.

Hyperechogenic linear zones in tumor formation were displayed simultaneously with the introduction of contrast in the medulla. Hypocontrasted area was visualized in the renal hilum (20 sec). The tumor formation was clearly visible (29 $\mathrm{sec}$ ) with slow entering of contrast from the periphery to the center. The inner parts of the formation were heterogeneous with hypercontrasted external contour (1:08) - (Fig. 3). The contrast was removed from the cortex and medulla, remained visible hypoechogenic formation with hypocontrasted contour. The different parts of the time-intensity curve of the tumor and kidney parenchyma were similar (Fig. 4) The diagnosis from CEUS was angiomyolipoma.

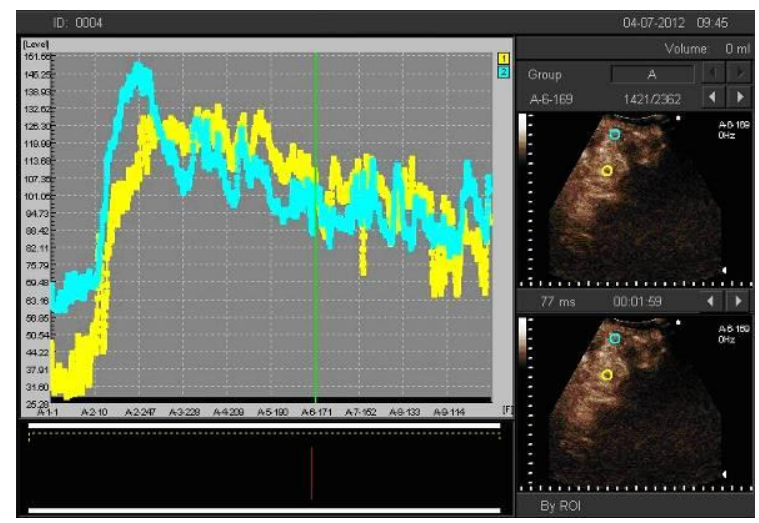

Figure 4: CEUS - time intensity curve shows uniform contrast distribution in the tumor and the adjacent parenchyma.

The controvesrial data from the conventional ultrasound and CEUS urged contrast computed tomography $(\mathrm{CT})$, revealing preserved kidney sizes and drainage. In the right upper kidney pole rounded, active and inhomogeneous contrasted formation 31 $\mathrm{mm}$ was visualized. No enlarged lymph nodes along the lymphatic collectors were detected. The described formation had the morphological characteristics of renal cell carcinoma (Fig. 5).

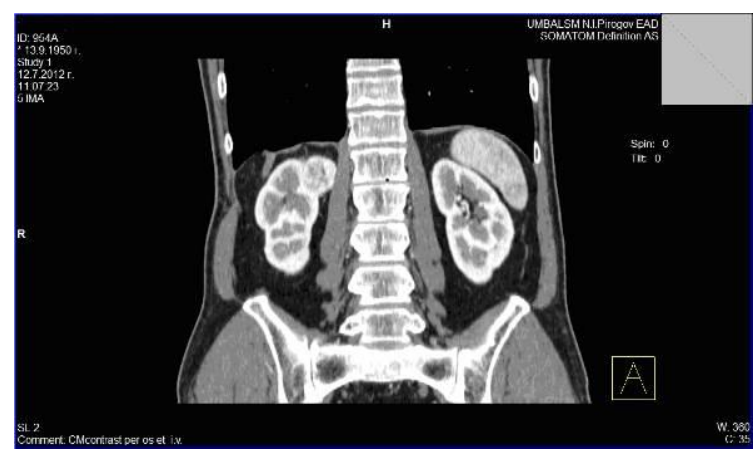

Figure 5: Computed tomography - the picture shows tumor in the right kidney.

The patient was referred to the Department of Urology, where he underwent right-sided nephrectomy - the histological result was clear-cell renal carcinoma, nuclear polymorphism Furman 1, with zone of cystic degeneration. The renal hilar fat and veins showed no engagement. There was epithelioid cell granuloma with single Langhans giant cells 
among the tumor and in the hilar fat. Several granulomas revealed with caseous necrosis (Fig. 6). The T-SPOT.TB was performed revealing the presence of reactive specific effector T-cells to the antigen on CEF 10 Mycobacterium tubercolosis.

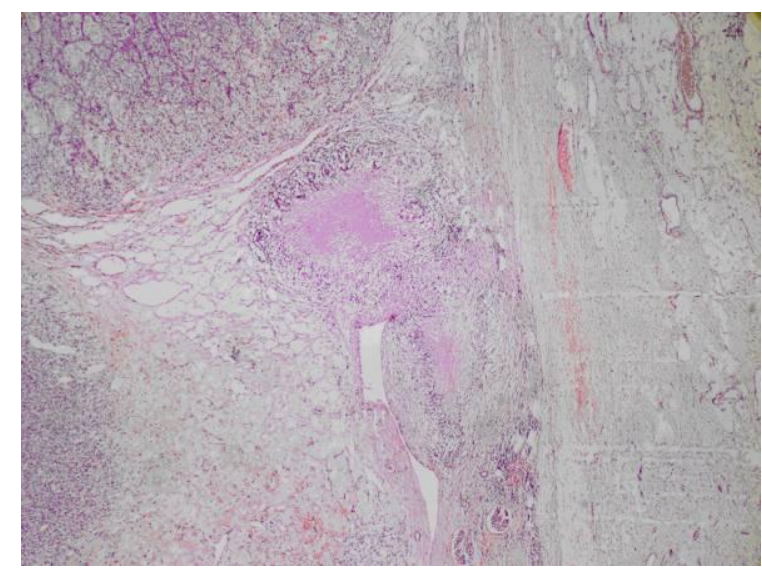

Figure 6: Histological result - the picture shows epithelioid cell granuloma with caseous necrosis.

\section{Discussion}

Granulomatous inflammation is characterized by the accumulation of epitheloid histiocytes and multinucleated giant cells [1]. The cause of granuloma formation can be infectious (tuberculosis, schistosomiasis, blastomycosis, etc.), non-infectious (Crohn's disease, Wegener's granulomatosis, sarcoidosis, granuloma - a type of foreign body, tumors). Granulomas have been described in the stroma of malignant tumors of breast carcinoma, colon carcinoma, seminoma, Hodgkin's lymphoma, and others. Tumor associated granulomatous reaction may be due to: 1) T-cell mediated response against antigens expressed by the tumor (immunologic mechanism for the formation of tumor associated granulomas), 2) chronic inflammatory reaction - a specific process such as tuberculosis. Granulomatous response in renal cell carcinoma is rare. Coexistence of tuberculosis and neoplasm in the same organ is rare, particularly in the kidney [2, 3].

Formation of granulation tissue around the necrosis is typical (but not specific) for tuberculous granuloma (tubercle). The granuloma contains large amounts of epitheloid cells, Langhans-type giant cells, lymphocytes, single plasma cells and macrophages. There are no blood vessels in granuloma.

Despite the certain ultrasonography data of malignant tumor (the heterogenity of the formation, neovascularisation and irregular Doppler curve) our initially contrast enhanced ultrasound diagnosis was angiomyolipoma (slow entering of contrast agents from the periphery to the center of formation, continuously wash out phase. Time intensity curve in tumor formation had the same intensity as in the adjacent area) $[4,5]$. Probably slow uptake of contrast in the formation and continously wash out phase was due to the presence of granulomatous changes and caseous necrosis. The described hypoechogenic zone towards the hilum could be zone of caseous necrosis (no vessels which prevents contrasts). The presence of hyperechogenic linear zones might be the result of expansion of the fibrous tissue, or the presence of calcification in tumor formation.

For accurate diagnosis and determining the most appropriate therapeutic approach for suspected tumor is necessary combination of complex imaging triplex ultrasound, CEUS, CT and histological examination of suspected formation.

\section{References}

1. Narasimhaiah D, Manipadam $M$, Aswathaman $K$ Krishnamoorthy S. Renal cell carcinoma associated with granulomatous reaction. Saudi $J$ Kidney Dis Transpl. 2011;6:1211-1214.

2. Ouellet S, Albadine R, Sabbagh R. Renal cell carcinoma associated with peritumoral sarcoid-like reaction without intratumoral granuloma. Diagnostic Pathology. 2012;7:28.

3. Kumar V, Abbas AK, Fausto N. Acute and Chronic inflammation. In: Pathologic Basis of Disease. Saunders. 2006;7:47-86.

4. Tsocheva T, Djerassi R. Diagnostic possibilities of contrast enhanced ultrasound in diagnostic of urogenital tumors. Diagnostic and Therapeutic Ultrasound. 2012;20:8-14.

5. Xu ZF, Xu HX, Xie XY, Guang Y, Zheng YL, Lu M. Renal cell carcinoma and renal angiomyolipoma: differential diagnosis with real-time contrast-enhanced ultrasonography. J Ultrasound Med. 2010;29:709-717. 